\title{
A One Pot Green Synthesis and Characterisation of Iron Oxide-Pectin Hybrid Nanocomposite
}

\author{
Foba-Tendo J. Ngenefeme ${ }^{*}$, Namanga J. Eko ${ }^{1}$, Yufanyi D. Mbom ${ }^{1}$, Ndinteh D. Tantoh ${ }^{2}$, \\ Krause W. M. Rui ${ }^{2}$
}

${ }^{1}$ Department of Chemistry, Faculty of Science, University of Buea, Buea, Cameroon; ${ }^{2}$ Department of Chemistry, Rhodes University, Grahamstown, South Africa.

Email: ${ }^{*}$ jnfoba@yahoo.com

Received August $2^{\text {nd }}, 2012$; revised September 10 ${ }^{\text {th }}, 2012$; accepted October $10^{\text {th }}, 2012$

Copyright (C) 2013 Foba-Tendo J. Ngenefeme et al. This is an open access article distributed under the Creative Commons Attribution License, which permits unrestricted use, distribution, and reproduction in any medium, provided the original work is properly cited.

\begin{abstract}
Nano-composites comprised of magnetite nanoparticles in a pectin matrix were prepared by the co-precipitation method. Both sodium hydroxide and ammonium hydroxide were used as precipitating agents and the effects of stoichiometric ratios of iron salts to polymer concentration on particle properties were investigated. The precipitates obtained with $\mathrm{NH}_{4} \mathrm{OH}$ were sponge-like. The XRD pattern revealed magnetite as the only iron oxide phase present when $\mathrm{NH}_{4} \mathrm{OH}$ was used as precipitating base whilst nanoparticles precipitated using $\mathrm{NaOH}$ showed the presence of additional peaks. The nanocomposites obtained were further characterized using transmission electron microscopy (TEM), Fourier transform infrared spectroscopy (FT-IR), scanning electron microscopy (SEM), X-ray powder diffraction (XRD), and zeta-potential. FT-IR confirmed the COO-Fe linkage. Zeta-potential analysis showed a potential change from positive in pure magnetite to negative in polymer coated magnetite depending on the oxide-polymer ratio. SEM and TEM showed fairly evenly distributed nanosize pores.
\end{abstract}

Keywords: Co-Precipitation Method; Magnetite; Pectin; Zeta Potential; Nanocomposite

\section{Introduction}

Current and growing interest in nanostructures results from their numerous potential applications such as in materials development, biomedical sciences, electronics, optics, magnetism, energy storage, and electrochemistry [16]. Nano-sized materials display properties that differ from their respective bulk counterparts [7]. Magnetic nanomaterials constitute one of the most interesting classes because of current and potential biological, biomedical and environmental applications like their use as adsorbents for the removal of dyes from polluted waters and the heavy metal uptake in water treatment procedures [8-10]. The low toxicity, biocompatibility, cost-effectiveness and high surface area to volume ratios, as a function of particle size, when combined with their ability for surface chemical modification, represent major advantages compared to other nanoparticles $[3,8,11]$. Maghemite and magnetite are the two main forms of iron oxide nanoparticles with diverse applications. Successful approaches to the

*Corresponding author. synthesis of stable magnetic nanoparticles use capping/ stabilisation agents which may be simple molecules or polymeric. Among the polymeric capping agents, biopolymers are of special interest due to their biocompatibility and biodegradability.

Pectin is a group of complex polysaccharides that contain 1,4-linked $\alpha$-D-galacturonic acid [12]. Pectin has a very complex structure that depends on both its source and the extraction process. Its properties depend on its degree of esterification [13]. Pectin has been used extensively in the food, cosmetics, pharmaceutical and biomedical industries for their gel forming properties in the presence of multivalent cations. Pectin is biodegradable, biocompatible and is reported to be bioactive [14]. Pectin also contains the hydroxyl (-OH) functional group which enhances its chemical properties and $\mathrm{COOH}$ groups which permit direct bonding to $\mathrm{Fe}_{3} \mathrm{O}_{4}$.

Co-precipitation and high temperature solution phase methods have been the two most widely used methods, in the synthesis of iron oxide nanoparticles. The high temperature solution phase method involves the decomposi- 
tion of iron organic precursor $\mathrm{Fe}(\mathrm{acac})_{3}, \mathrm{Fe}(\mathrm{CO})_{5}$, etc. in an organic solvent such as hexane or alkyl ether in the presence of a mild reducing agent such as polyol or hydrazine $[15,16]$. Iron oxide obtained through this method finds very little application in biological, environmental and biomedical fields because of the use of toxic organic solvents during synthesis. Furthermore, magnetic NPs synthesized in organic solvents for biological applications must be surface treated in order to solubilise them in aqueous solutions. The coprecipitation method involves the co-precipitation of ferric and ferrous ions using a base such as sodium hydroxide $(\mathrm{NaOH})$ or ammonium hydroxide $\left(\mathrm{NH}_{4} \mathrm{OH}\right)$ in an aqueous medium [17]. Even though, the high temperature method has been proven to be more efficient in synthesizing mono-dispersed iron oxide nanoparticles [18], the co-precipitation method has gained favour due to the use of low temperature and environmentally friendly reagents and conditions.

The synthetic procedures for magnetite nanoparticles in literature report the formation of both magnetite and maghemite. This is due to rapid conversion of magnetite to maghemite under oxidizing environments such as air. The stability of magnetite has however been improved by coating with different materials depending on the area of application [19].

Stabilisation of magnetite nanoparticles with a polymer is achieved in two ways; either by synthesis of magnetite NPs in the presence of the polymer (a one pot synthesis) or the magnetite NPs are synthesized in one reaction and then coated with a polymeric material in an entirely different reaction. Synthetic polymers, such as polyethylene glycol, have been reported to stabilize magnetite NPs [20,21]. Stabilization of magnetite NPs by coating them with non-toxic polymers, in turn reduces the toxicity of magnetite in living systems. Biological or natural polymers are now gaining more interest as suitable polymeric materials for coating magnetite NPs since they are non-toxic.

Generally, the use of naturally occurring polymers as stabilising/capping agents for magnetite is regarded as a green chemistry approach [20]. Polysaccharides are receiving increasing interest in the green chemistry approach to stabilize/coat magnetic nanoparticles. A green synthetic strategy of oxide NPs (including magnetite) where starch was used as the stabilizing agent has been reported [22]. Alginates have been reported to act as a scaffold for the synthesis of magnetite NPs [23]. A modified chitosan (chitosan-polyacrylic acid) was used to improve the binding on magnetite NPs. The use of a modified chitosan (carboxylmethyl-chitosan) as a stabilizing/coating agent in the synthesis of magnetite has also been reported [17].

In this paper, we present a simpler and environmentally friendly approach to producing magnetite-polymer hybrid nanocomposites with closer control over particle size, size distribution, and hopefully, a more uniform morphology by a choice of the precipitating base and the manipulation of oxide-polymer ratio. The approach uses pectin, a naturally occurring polymer, without any modification of polymer, as a stabilizing agent. The magnetitepolymer nanocomposites will be compared to iron oxides obtained using $\mathrm{NaOH}$ as precipitating base.

The synthesis of $\mathrm{Fe}_{3} \mathrm{O}_{4}$ using the co-precipitation method in the presence of pectin is expected to initiate the covalent linkage instantaneously which should prevent further particle growth, resulting in pectin-coated ferrite composites with closer control over particle size.

\section{Experimental}

\subsection{Materials}

Ferric chloride $\left(\mathrm{FeCl}_{3} \cdot 6 \mathrm{H}_{2} \mathrm{O}\right)$, ferrous chloride $\left(\mathrm{FeCl}_{2} \cdot 4 \mathrm{H}_{2} \mathrm{O}\right)$ and all the other chemicals were of reagent grade and were used as such. Sodium hydroxide pellets and ammonia solution were used as precipitating agents while citrus pectin $(\mathrm{DE}=76 \%)$ was used as the stabilizer/coating agent. Distilled water was used throughout the synthesis and the entire process was carried out at room temperature under an inert atmosphere initiated by the flow of nitrogen gas. The iron salts were stored under nitrogen gas in a dessicator; both sodium hydroxide solution $(1.5 \mathrm{M}, 500 \mathrm{~mL})$ and ammonium hydroxide $(1.5 \mathrm{M}$, $500 \mathrm{~mL}$ ) were deoxygenated by the flow of nitrogen gas for at least 30 minutes prior to use.

\subsection{Methods}

Pectin solutions of different concentrations $(\% \mathrm{~W} / \mathrm{V})$, as indicated in Table 1, were prepared by dissolving givenmasses of it in $250 \mathrm{~mL}$ distilled water, under agitation, in a three neck reaction flask. The reaction flask was deoxygenated for 30 minutes and a $50 \mathrm{~mL}$ solution of a 2:1 molar ratio of ferric and ferrous ions was added drop wise into the pectin solution under stirring. The volume of solution was maintained at $300 \mathrm{~mL}$ and the reaction medium was further deoxygenated for an additional 20 minutes. Addition of the iron salts resulted in the formation of a brown colour. The base was then added dropwise

Table 1. Reaction conditions and parameters.

\begin{tabular}{cccccc}
\hline Sample & $\begin{array}{c}\mathrm{Fe}^{3+} / \\
\mathrm{Fe}^{2+}\end{array}$ & $\begin{array}{c}\text { Mass of } \mathrm{Fe}^{3+} \\
\text { salt }(\mathrm{g})\end{array}$ & $\begin{array}{c}\text { Mass of } \mathrm{Fe}^{2+} \\
\text { salt }(\mathrm{g})\end{array}$ & $\begin{array}{c}\text { Pectin Conc. } \\
(\% \mathrm{~W} / \mathrm{V})\end{array}$ & $\begin{array}{c}\text { Precipitating } \\
\text { Agent }\end{array}$ \\
\hline PIO-0 & $2: 1$ & 10.4 & 4.0 & 0.0 & $\mathrm{NH}_{4} \mathrm{OH}$ \\
PIO-1 & $2: 1$ & 7.8 & 3.0 & 0.3 & $\mathrm{NH}_{4} \mathrm{OH}$ \\
PIO-2 & $2: 1$ & 7.8 & 3.0 & 0.3 & $\mathrm{NaOH}$ \\
PIO-3 & $2: 1$ & 7.8 & 3.0 & 0.5 & $\mathrm{NH}_{4} \mathrm{OH}$ \\
\hline
\end{tabular}


using a syringe till the solution became completely black indicating the formation of magnetite. The reaction conditions and the variation in parameters are presented in Table 1.

The resultant black residue obtained was filtered and washed several times with distilled water until the $\mathrm{pH}$ dropped to 7 . The black precipitate was then freeze-dried and characterized.

\section{Results and Discussions}

The effect of the precipitating agents sodium hydroxide and ammonium hydroxide on the texture of the particles formed are summarized in Table 2.

When $\mathrm{NaOH}$ is used as base the solution has a high $\mathrm{pH}$ of 13 . At this $\mathrm{pH}$, the polymer is de-esterified and depolymerised during the co-precipitation process and hence powders are obtained.

The exclusive production of powders when $\mathrm{NaOH}$ is used could be attributed to the depolymerisation of pectin chains by the stronger base. The retention of the gel structure when $\mathrm{NH}_{4} \mathrm{OH}$ is used indicates that the chain structure of the pectin polymer remained largely intact, suggesting limited depolymerisation.

The differences in $\mathrm{pH}$ evolution can be attributed to the differences in the influence of the base on pectin. $\mathrm{NH}_{4} \mathrm{OH}$ will neutralise free carboxylic acid groups in the process of amidation. $\mathrm{NH}_{4} \mathrm{OH}$ is however a weak base to cause the depolymerisation of the pectin. $\mathrm{NaOH}$ additions will neutralise the $\mathrm{COOH}$ groups, cause de-methyllation and depolymerisation. The plateau in increase of $\mathrm{pH}$ corresponds to the de-methylation phase, after the initial neutralisation of $-\mathrm{COOH}$ groups in the pectin.

Pectin strongly binds both iron(III) and iron(II) ions through a COO- linkage. NP-gel composites obtained at lower pectin content suggests that insufficient pectin was available to bind with iron(III) ions.

\subsection{Powder X-Ray Diffraction (XRD) Analysis}

The XRD patterns for the samples are shown in Figure 1. Figures 1(a), (b) and (d) have diffraction peaks that are consistent with the seven diffraction peaks at (2 20$)$, (3 1 1), (4 00 ), (4 $\left.\begin{array}{lll}4 & 2\end{array}\right),\left(\begin{array}{lll}5 & 1 & 1\end{array}\right)$, and (4 $\left.4 \begin{array}{ll}4 & 0\end{array}\right)$ by comparison with Joint Committee on Powder Diffraction Standards

Table 2. Effect of base on the texture of the particles.

\begin{tabular}{lcc}
\hline Samples & Base used as precipitation agent & Texture of product \\
\hline PIO-0 & $\mathrm{NH}_{4} \mathrm{OH}$ & Powder \\
PIO-1 & $\mathrm{NH}_{4} \mathrm{OH}$ & Powder plus gel-network \\
PIO-2 & $\mathrm{NaOH}$ & Powder \\
PIO-3 & $\mathrm{NH}_{4} \mathrm{OH}$ & Gel network \\
\hline
\end{tabular}

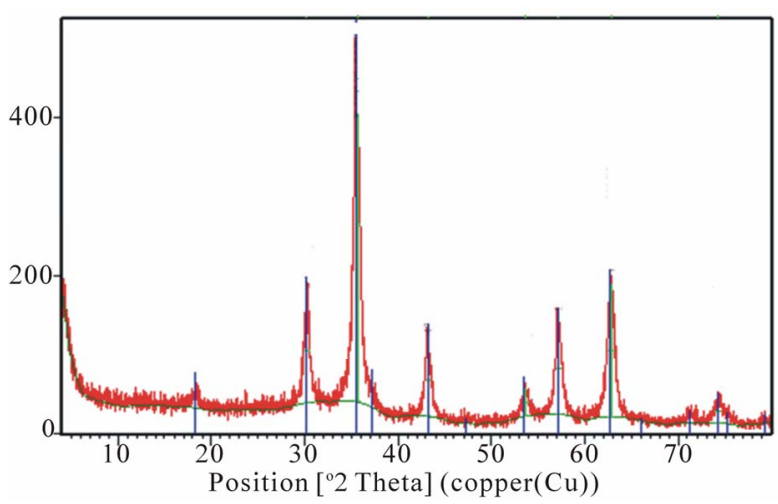

(a)

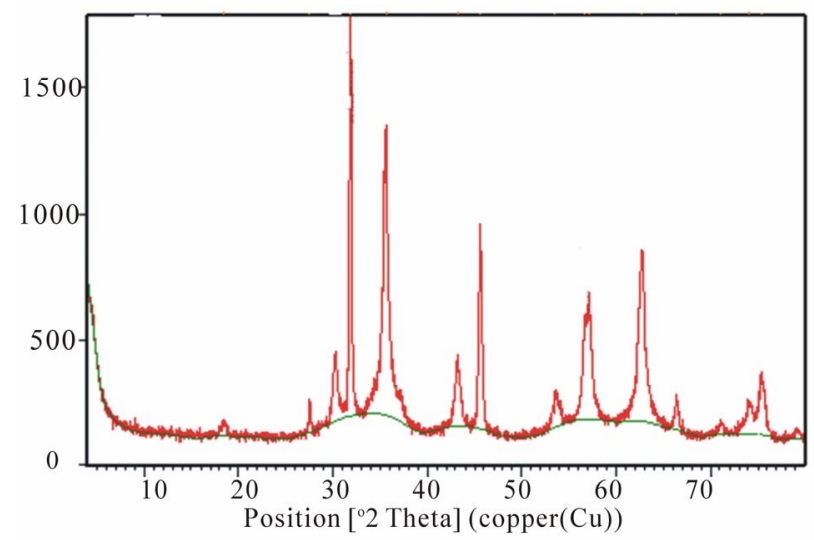

(b)

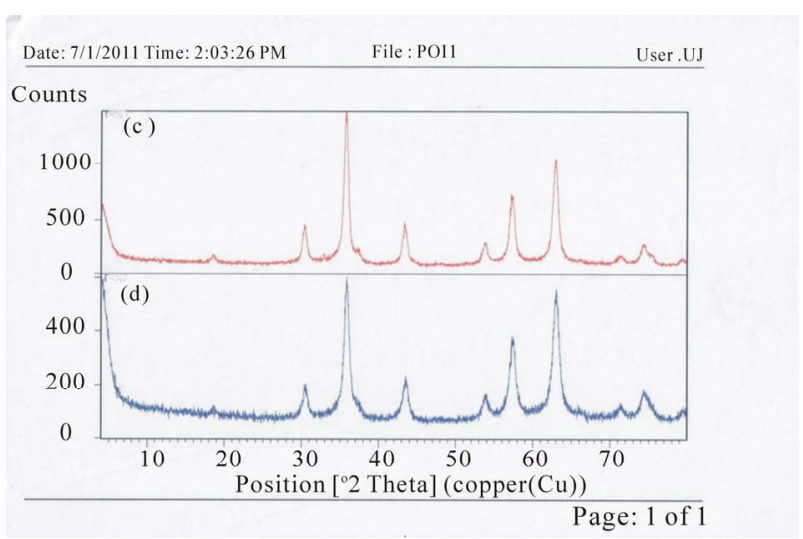

Figure 1. XRD spectra for (a) PIO-0; (b) PIO-2; (c) PIO-1; and (d) PIO-3.

(JCPDS card, file No. 790418), which are indexed to the cubic spinel phase of $\mathrm{Fe}_{3} \mathrm{O}_{4}$. It reveals that the resultant nanoparticles in samples PIO-0, PIO-1 and PIO-3 are $\mathrm{Fe}_{3} \mathrm{O}_{4}$. Figure 1(b) had more diffraction peaks than the other samples which suggest the formation of a mixed iron oxide phase. The particle sizes were quantitatively evaluated from the XRD data using the Debye-Scherrer equation,

$$
D=\frac{k \lambda}{\beta \cos \theta}
$$


where $k$ is Scherrer constant (0.89), $\lambda$ the X-ray wavelength $(0.15405 \mathrm{~nm}), \beta$ the peak width of half-maximum, and $\theta$ is the Bragg diffraction angle. The d-spacing of the (311) diffraction of all the samples was in the range $2.52-2.53 \AA$. The average particle sizes were found to decrease with increase in polymer concentration as shown in Table 3.

The extra peaks in the XRD pattern of PIO-2 suggest the presence of another form of iron oxide (preferably maghemite) as a result of using a strong base $(\mathrm{NaOH})$ as precipitating agent.

\subsection{Fourier Transform Infrared (FT-IR) Spectra}

In the spectrum of the pure pectin sample (Figure 2), the peaks at $3253 \mathrm{~cm}^{-1}$ and $2946 \mathrm{~cm}^{-1}$ represent secondary hydroxyl groups and carboxylic hydroxyl groups, respectively. Also, the peak at $1737 \mathrm{~cm}^{-1}$ is a characteristic peak of pectin (representing the carbonyl of the esterified pendant group). The intense peak at $1015 \mathrm{~cm}^{-1}$ arises from the glycosidic bonds linking two galacturonic sugar units [10]. Comparing Figures 3-5 to Figure 2, the two new peaks at $1586-1587 \mathrm{~cm}^{-1}$ and $1392-1394 \mathrm{~cm}^{-1}$ have been attributed to the symmetric and asymmetric

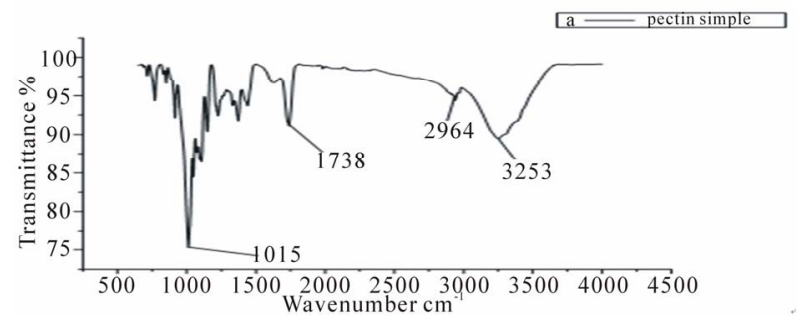

Figure 2. IR spectrum of pure pectin.

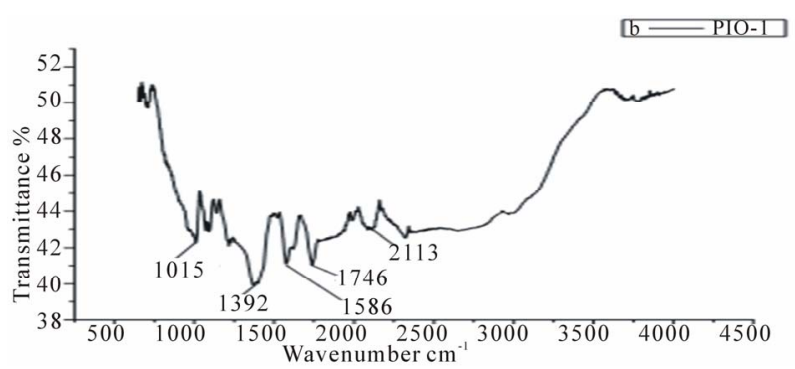

Figure 3. IR spectrum of PIO-1.

Table 3. Particle sizes based on the dedye-scherrer equation.

\begin{tabular}{cc}
\hline Sample & Average particle size \\
\hline PIO-0 & $15 \mathrm{~nm}$ \\
PIO-1 & $13 \mathrm{~nm}$ \\
PIO-3 & $9 \mathrm{~nm}$ \\
\hline
\end{tabular}

stretching of carboxylate-metal (COO-Fe) linkage [10, 24]. Reduction in peak intensity at $1017 \mathrm{~cm}^{-1}$ in Figure 4 results from the breaking of glycosidic bonds, hence, depolymerization of the pectin, while reduction in peak intensity at $1746 \mathrm{~cm}^{-1}$ is as a result of significant de-esterification of the ester pendant group of the pectin polymeric chain $[25,26]$. These FT-IR results support the suggestion that $\mathrm{NaOH}$ causes significant deploymerisation of pectin whilst $\mathrm{NH}_{4} \mathrm{OH}$ does not.

\subsection{Zeta Potential Analysis}

The Zeta potential values for the samples are represented graphically in Figure 6.

The surface charge of magnetite prepared in the absence of the coating agent was positive $(+26 \mathrm{eV})$ which implies the presence of iron ions at the surface of the $\mathrm{Fe}_{3} \mathrm{O}_{4}$ particles. Sample PIO- 1 had a positive value $(+22$ $\mathrm{eV}$ ) contrary to the expected negative value since FT-IR analysis revealed the COO-Fe linkage. This result indicates that, not all the $\mathrm{Fe}_{3} \mathrm{O}_{4}$ particles were coated which could be due to the high concentration of magnetite particle

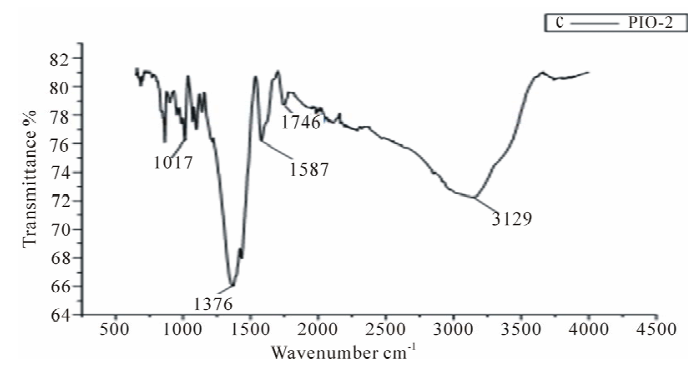

Figure 4. IR spectrum of PIO-2.

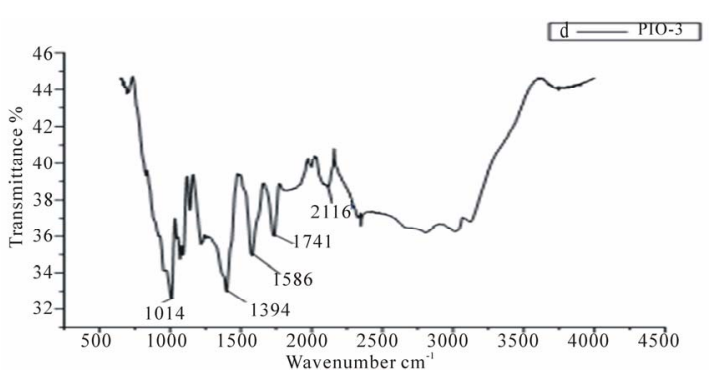

Figure 5. IR spectra of PIO-3.

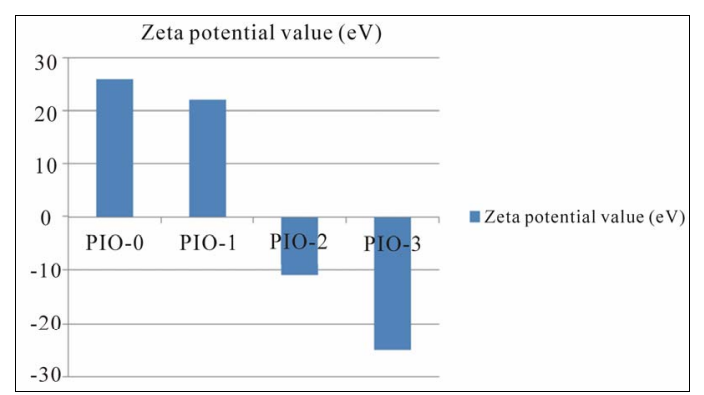

Figure 6. Graphical representation of zeta potential values. 
to polymer ratio. This observation is in agreement with the texture (mixed powder and gel) of PIO-1. Sample PIO-2 which had the same magnetite/polymer ratio as sample PIO-1 however gave a negative value of $-11 \mathrm{eV}$. The difference in potential of sample PIO-1 and PIO-2 can be attributed to the different bases used during the co-precipitation process. In PIO-1 where ammonium hydroxide was used as the base, some of the esterified groups were converted to the amide group instead of carboxylic group due to the presence of the ammonium ion. In PIO2 where sodium hydroxide was used as the base, demethylation at alkaline $\mathrm{pH}$ gave rise to carboxylate ions. Some of these ions bind to $\mathrm{Fe}_{3} \mathrm{O}_{4}$ particles through linkages with $\mathrm{Fe}$ cations. The negative higher potential values for PIO-3 indicate more complete coating of iron surface cations through $\mathrm{O}-\mathrm{Fe}$ and $\mathrm{N}-\mathrm{Fe}$ linkages. The surface properties of these hybrid composites could thus be tuned by simply changing the oxide-polymer ratio.

\subsection{Thermogravimetric Analysis (TGA)}

Samples PIO-0 and PIO-3 were analyzed by TGA to investigate the percent polymer and percent magnetite. The thermal behaviour of the samples is shown in Figure 7. TGA of pure magnetite showed no significant change in mass with increase in temperature, while magnetite coated with pectin showed a mass drop between $200^{\circ} \mathrm{C}$ and

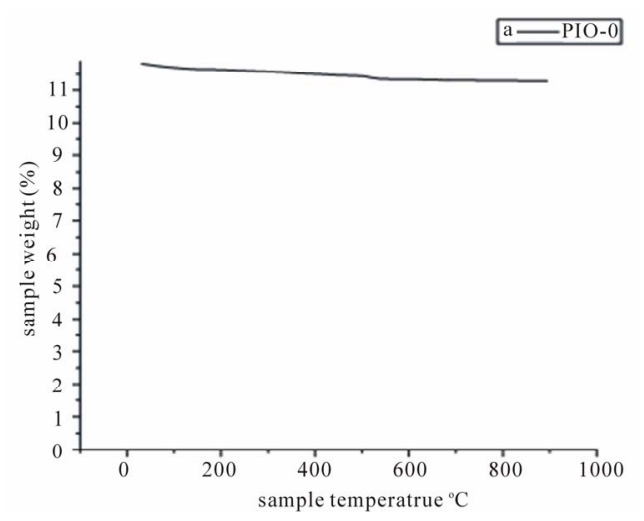

(a)

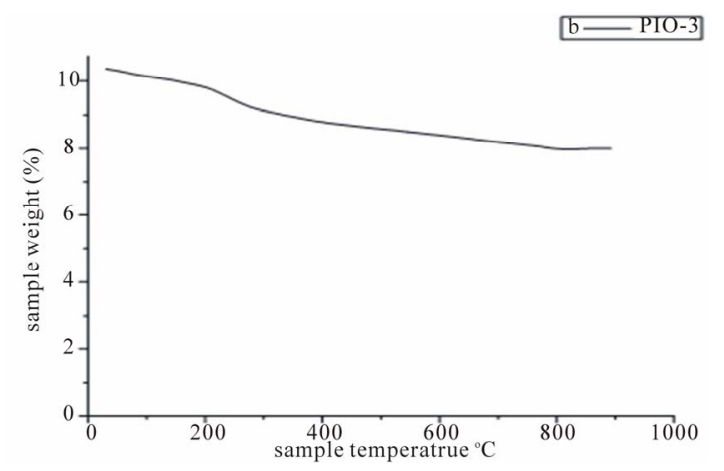

(b)

Figure 7. TGA spectra of (a) PIO-0 and (b) PIO-3. $350^{\circ} \mathrm{C}$. This mass drop might be attributed to the thermal degradation of the pectin coating on the nanoparticles. Sample PIO-3 had $22.95 \%$ polymer and $77.05 \%$ magnetite.

\subsection{Scanning Electron Microscopy (SEM)}

The surface morphology of the selected samples (PIO-2 and PIO-3), analyzed using Joel-Jem 2100 electron microscope, are presented in Figure 8.

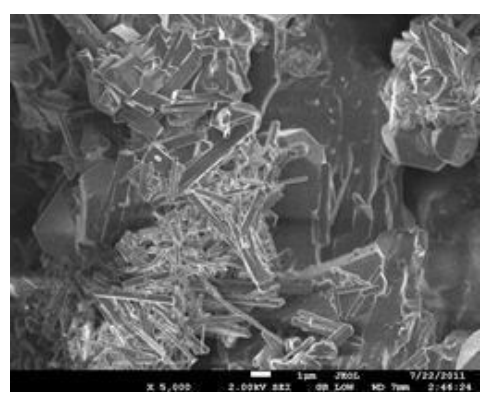

(a)

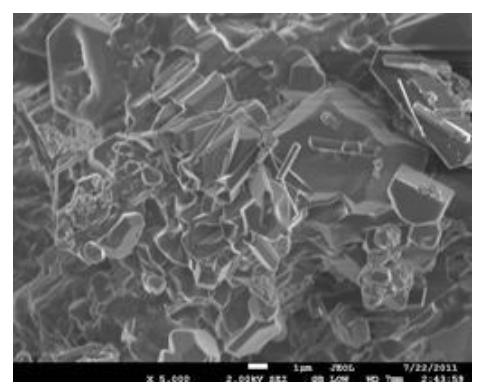

(b)

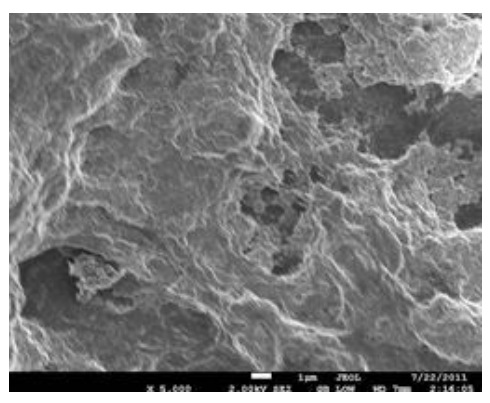

(c)

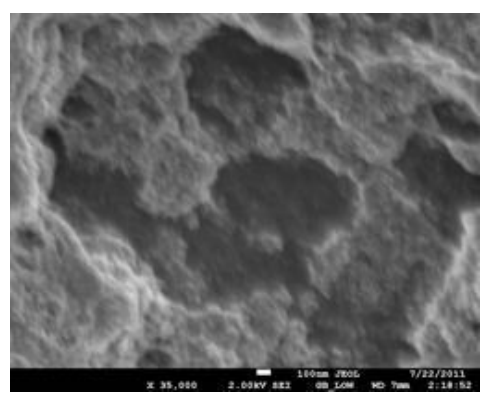

(d)

Figure 8. (a) and (b) SEM images of PIO-2 (NaOH was used as precipitating base); (c) and (d) SEM images of PIO-3 ( $\mathrm{NH}_{4} \mathrm{OH}$ was used as the precipitating base). 
Figures 8(a) and (b) show flat-faced particles of varied shapes and sizes for precipitates obtained with $\mathrm{NaOH}$. The particle like nature of these precipitates indicates a non-polymeric coating of $\mathrm{Fe}_{3} \mathrm{O}_{4}$ particles, which suggests that $\mathrm{NaOH}$ depolymerised the pectin chain during the coprecipitation process. The particle-like nature of these precipitates contrasts sharply with the foam-like material formed when $\mathrm{NH}_{4} \mathrm{OH}$ is used as precipitating agent. Figures 8(c) and (d) show a foam-like material indicating the retention of a polymeric coating around the oxide particles when $\mathrm{NH}_{4} \mathrm{OH}$ is used as base. These observations again suggest that the pattern of hydrolysis is different for the two alkalis. The foam-like appearance as well as the finer particles obtained indicate the retention of large chain pectin forms whilst their free particles indicate their absence in $\mathrm{NaOH}$ precipitated oxides. These observations are consistent with previous studies on the

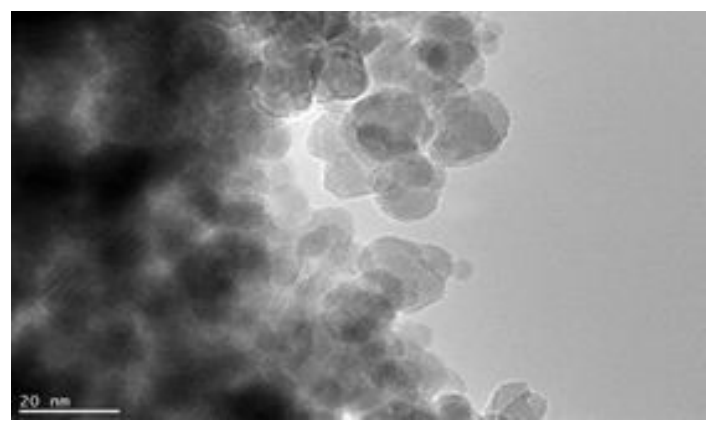

(a)

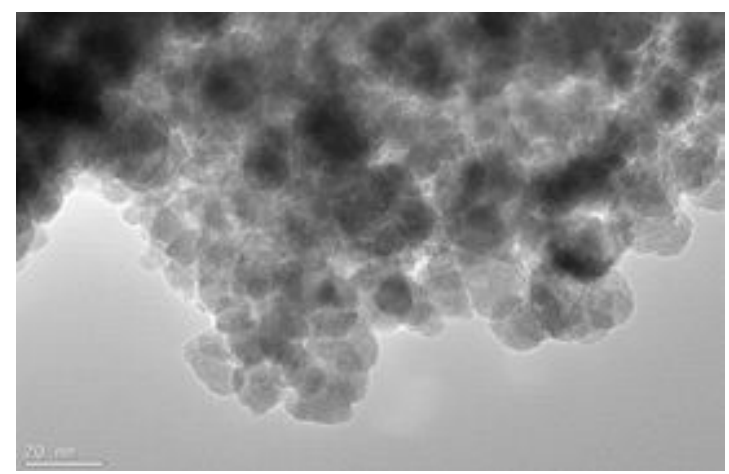

(c)

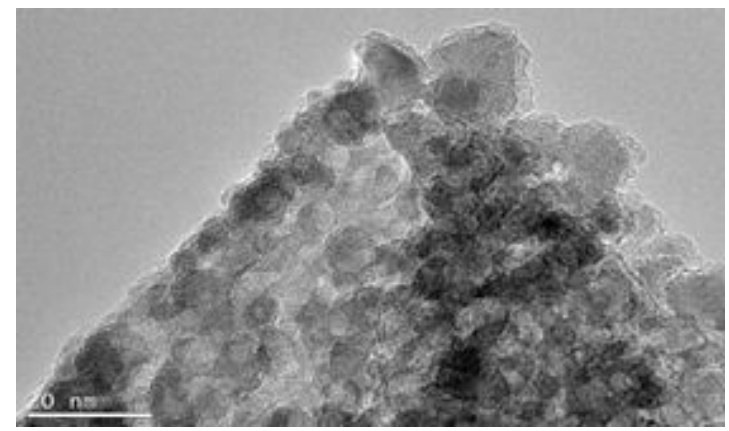

(e) influence of $\mathrm{NaOH}$ and $\mathrm{NH}_{4} \mathrm{OH}$ on demethylation and glycosidic hydrolysis of pectins.

\subsection{High Resolution Transmission Electron Microscopy (HR-TEM)}

The magnetite particle sizes, the polymer coating nature and dispersion of magnetite particle within the polymer matrix were examined using Joel JSM 7500F electron microscope. Figures 9(a) and (b) show the TEM images for pure magnetite which display the largest particle size distribution with evidence of aggregation of magnetite magnetic particles. Figures 9(c) and (d) show incomplete polymer coating of magnetite in sample PIO-1 as many parts of the analyzed section revealed magnetite particles at the surface. This is consistent with results obtained from Zeta potential analysis of PIO-1. Figures 9(e)

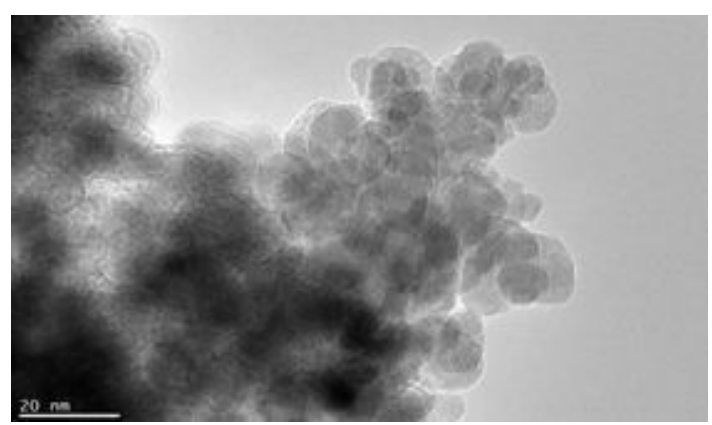

(b)

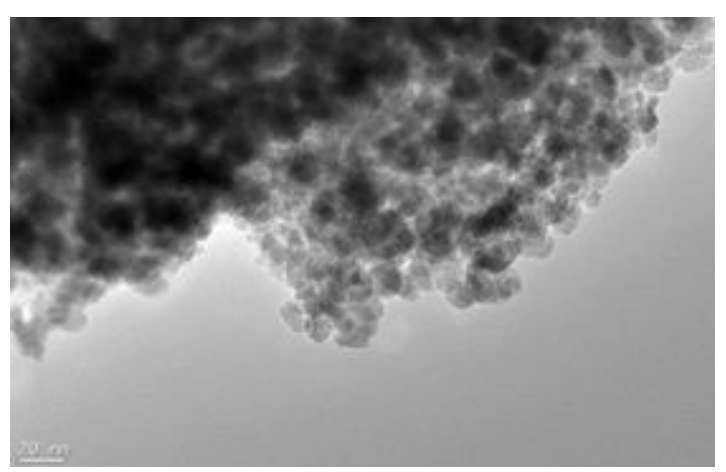

(d)

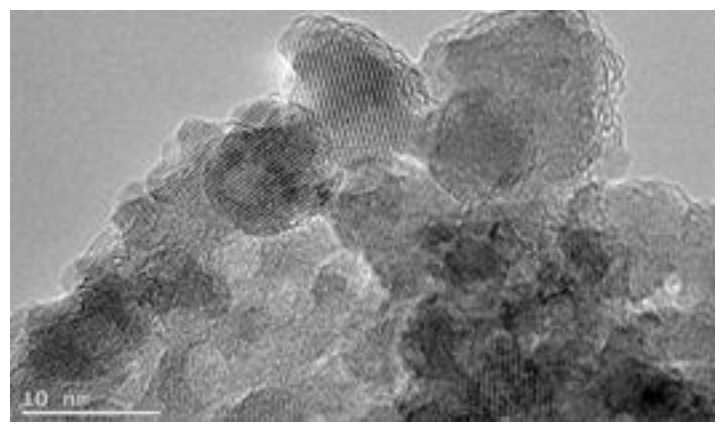

(f)

Figure 9. TEM images: (a) and (b) PIO-0; (c) and (d) PIO-1; and (e) and (f) PIO-3. 
and (f) show complete polymer coating of magnetite nanoparticles. TEM analysis demonstrates that the presence of pectin reduces the size distribution of the magnetite nanoparticles and prevents particle aggregation due to the dispersion of magnetite within the polymer matrix. The magnetite nanoparticle sizes were found to be consistent with the results from XRD analysis. These TEMs still show some level of aggregation. They also show that the coating of the oxide particles is not uniform. A close look at PIO-1 and PIO-3 indicates there is some improvement in the dispersion of oxide particles in the polymer matrix with higher pectin-to-metal ratio.

\section{Conclusion}

Hybrid nanocomposites comprising magnetite nanoparticles in a pectin matrix have been synthesised in a simple, room temperature one-step synthetic procedure using ammonium hydroxide as precipitating agent. Control of magnetite particle size and particle size distribution improved with increasing pectin content, which stabilized/coated the magnetite nanoparticles. These preliminary results indicate that a judicious choice of metal ion to pectin ratio can permit a better control on particle size and size distribution and also presents itself as another approach at controlling the surface properties of the composite. The base used as precipitating agent coupled with the effect of depolymerisation lead to the probable in situ generation of oligomers. These oligomers acted rather as surfactants binding to the higher energy surfaces of the magnetite and/or maghemite crystal faces and as a result particle growth continued in one direction (that with the lowest surface energy) with the consequent formation of nanorods. The pectin-magnetite nanocomposites can possibly find application as fillers in magnetic tapes and magnetic fluids where dispersion is better, as templates for synthesis, as targeted drug delivery systems as well as in molecular separation and ion exchange. They can also find application in pollution control as adsorbents for cationic pollutants.

\section{Acknowledgements}

Dr. Jaco of Nelson Mandela Metropolitan University, South Africa for TEM analysis.

\section{REFERENCES}

[1] G. J. Connolly, Q. A. Pankhurst, S. K. Jones and J. Dobson, "Applications of Magnetic Nanoparticles in Biomedicine," Journal of Physical D: Applied Physics, Vol. 36, No. 13, 2003, pp. 167-181. doi:10.1088/0022-3727/36/13/201

[2] M. Christof and A. Niemeyer, "Nanoparticles, Proteins, and Nucleic Acids Biotechnology Meets Materials Science," Angewandte Chemie International Edition, Vol. 40,
No. 22, 2001, pp. 4128-4158.

[3] D. Jana, H. Radim, A. Vojtech, K. Rene, S. Oldrich and H. Jaromir, "Preparation and Properties of Various Magnetic Nanoparticles," Sensors, Vol. 9, No. 4, 2009, pp. 2352-2362.

[4] J. Lodhia, G. Mandarano, N. J. Ferris, P. E. Franzer and S. F. Cowell, "Development and Use of Iron Oxide Nanoparticles (Part 1): Synthesis of Iron Oxide Nanoparticles for MRI," Biomedical Imaging and Intervention Journal, Vol. 6, No. 2, 2010, p. e12.

[5] L. Zhang, H. Rong and C. G. Hong, "Synthesis and Kinetic Shape and Size Evolution of Magnetite Nanoparticles," Materials Research Bulletin, Vol. 41, No. 2, 2006, pp. 260-267. doi:10.1016/j.materresbull.2005.08.024

[6] R. Hernandez, S. Javier, N. Aurora, A. E. Tiberio and M. Carmen, "Structural Organization of Iron Oxide Nanoparticles Synthesized Inside Hybrid Polymer Gels Derived from Alginate Studied with Small-Angle X-Ray Scattering," Langmuir, Vol. 25, No. 22, 2009, pp. 13212-13218. doi:10.1021/la902441s

[7] S. P. Gubin, Y. I. Spichkin, G. Y. Yurkov and A. M. Tishin, "Nanomaterial for High-Density Magnetic Data Storage," Russian Journal of Inorganic Chemistry, Vol. 47, No. 1, 2002, pp. 32-67.

[8] G. Grini, "Non-Conventional Low-Cost Adsorbents for Dye Removal: A Review," Bioresource Technology, Vol. 97, No. 9, 2006, pp. 1061-1085.

[9] N. Dalali, M. Khoramnezhad, M. Habibizadeh and M. Faraji, "Magnetic Removal of Acidic Dyes from Waste Waters Using Surfactant-Coated Magnetic Nanoparticles: Optimization of Process by Taguchi Method," IPCBEE, Vol. 15, 2011, pp. 89-93.

[10] J.-L. Gong, X.-Y. Wang, G.-M. Zeng, L. Chen, J.-H. Deng, X.-R. Zhang and Q.-Y. Niu, "Copper (II) Removal by Pectin-Iron Oxide Magnetic Nanocomposite Adsorbent," Chemical Engineering Journal, Vol. 185-186, 2012, pp. 100-107. doi:10.1016/j.cej.2012.01.050

[11] L. D. Ana, T. Trindade, J. G. Brian, F. O. C. Benilde, N. C. Rui and M. G. Ana, "In Situ Synthesis of Magnetite Nanoparticles in Carrageenan Gels," Biomacromolecules, Vol. 8, No. 8, 2007, pp. 2350-2357.

[12] C. Mollea, F. Chiampo and R. Conti, "Extraction and Characterization of Pectins from Cocoa Husks: A Preliminary Study," Food Chemistry, Vol. 107, No. 3, 2008, pp. 13531356.

[13] Ştefania, V. Silvia, P. Marcel and L. Cornelia, "Polysacharides Based on Micro- and Nanoparticles Obtained by Ionic Gelation and Their Applications as Drug Delivery Systems," Revue Roumaine de Chimie, Vol. 54, No. 9, 2009, pp. 709-718.

[14] B. R. Sharma, L. Naresh, N. C. Dhuldhoya, S. U. Merchant and U. C. Merchant, "An Overview on Pectins," Times Food Processing Journal, Vol. 23, No. 2, 2006, pp. 4451.

[15] J. Xie, P. Sheng, B. Nathan, P. Nader, X. W. Shan and S. Shouheng, "One-Pot Synthesis of Monodisperse Iron Oxide Nanoparticles for Potential Biomedical Applications," Pure Applied Chemistry, Vol. 78, No. 5, 2006, pp. 
1003-1014. doi:10.1351/pac200678051003

[16] Y. S. Lin and L. H. Christy, "Synthesis and Characterization of Biocompatible and Size-Tunable Multifunctional Porous Silica Nanoparticles," Chemical Materials, Vol. 21, No. 17, 2009, pp. 3979-3986. doi: $10.1021 / \mathrm{cm} 901259$ n

[17] H. A. S. Nurul, A. M. Pauzi and R. O. Mohammed, "Synthesis and Characterization of Carboxymethyl Chitosan$\mathrm{Fe}_{3} \mathrm{O}_{4}$ Nanoparticles," Prosiding Seminar Kimia Bersama, KM-ITB, 9-11 June 2009, pp. 291-301.

[18] S. Sun and H. Zeng, "Size-Controlled Synthesis of Magnetite Nanoparticles," Journal of the American Chemical Society, Vol. 124, No. 28, 2002, pp. 8204-8205. doi:10.1021/ja026501x

[19] C. Tassa, S. Y. Shaw and R. Weissleder, "Dextran-Coated Iron Oxide Nanoparticles: A Versatile Platform for Targeted Molecular Imaging, Molecular Diagnosis, and Therapy," Accounts of Chemical Research, Vol. 44, No. 10, 2011, pp. 842-852. doi:10.1021/ar200084x

[20] S. F. Chin, S. C. Pang and H. T. Ching, "Green Synthesis of Magnetite Nanoparticles (via Thermal Decomposition Method) with Controllable Size and Shape," Journal of Material and Environmental Science, Vol. 2, No. 3, 2011, pp. 299-302.

[21] A. Hua, F. Christopher, W. Brent, S. Xintao, D. P. Marty, F. David, D. Jeffrey and G. Jinming, "Magnetite-Loaded Polymeric Micelles as Ultrasensitive Magnetic-Resonance Probes," Advanced Materials, Vol. 17, No. 16, 2005, pp.
1949-1952. doi:10.1002/adma.200401904

[22] V. Diana, T. Alina, P. Greta, T. Madalina, P. Carmen, I. Adelina and C. Oana, "A Green Synthetic Strategy of Oxide Materials: Polysaccharides-Assisted Synthesis Part II. Starch-Assisted Synthesis of Nanosized Metal-Oxides," Revue Roumaine de Chimie, Vol. 55, No. 11-12, 2010, pp. 1017-1026.

[23] P. V. Finotelli, D. A. Sampaio, M. A. Morales, A. M. Rossi and M.H. Rocha-Leão, "Ca-Alginate as Scaffold for Iron Oxide Nanoparticles Synthesis," Brazilian Journal of Chemical Engineering, Vol. 25, No. 4, 2008, pp. 759-764. doi:10.1590/S0104-66322008000400013

[24] J.-F. Liu, Z. Shan, H. Zhao and G.-B. Jiang, "Coating $\mathrm{Fe}_{3} \mathrm{O}_{4}$ Magnetic Nanoparticles with Humic Acid for High Efficient Removal of Heavy Metals in Water," Environmental Science and Technology, Vol. 42, No. 18, 2008, pp. 6949-6954. doi:10.1021/es800924c

[25] M. A. Coimbra, A. Barros, M. Baros, D. N. Rutledge and I. Delgadillo, "Multivariate Analysis of Uronic Acid and Neutral Sugar in Whole Pectic Samples by FT-IR Spectroscopy," Carbohydrate Polymer, Vol. 37, No. 3, 1998, pp. 241-248. doi:10.1016/S0144-8617(98)00066-6

[26] R. Gnanasambandam and A. Proctor, "Determination of Pectin Degree of Esterification by Diffuse Reflectance Fourier Transform Infrared Spectroscopy," Food Chemistry, Vol. 68, No. 3, 2000, pp. 327-332. doi:10.1016/S0308-8146(99)00191-0 